\title{
Visual and central attention share a capacity limitation when the demands for serial item selection in visual search are high
}

\author{
Christina B. Reimer ${ }^{1,2}$ (D) Torsten Schubert ${ }^{1}$
}

Published online: 23 January 2020

(C) The Psychonomic Society, Inc. 2019

\begin{abstract}
Visual and central attention are limited in capacity. In conjunction search, visual attention is required to select the items and to bind their features (e.g., color, form, size), which results in a serial search process. In dual-tasks, central attention is required for response selection, but because central attention is limited in capacity, response selection can only be carried out for one task at a time. Here, we investigated whether visual and central attention rely on a common or on distinct capacity limitations. In two dualtask experiments, participants completed an auditory two-choice discrimination Task 1 and a conjunction search Task 2 that were presented with an experimentally modulated temporal interval between them (stimulus onset asynchrony [SOA]). In Experiment 1, Task 2 was a triple conjunction search task. Each item consisted of a conjunction of three features, so that target and distractors shared two features. In Experiment 2, Task 2 was a plus conjunction search task, in which target and distractors shared the same four features. The hypotheses for conjunction search time were derived from the locus-of-slack method. While plus conjunction search was performed after response selection in Task 1, a small part of triple conjunction search was still performed in parallel to response selection in Task 1. However, the between-experiment comparison was not significant, indicating that both search tasks may require central attention. Taken together, the present study provides evidence that visual and central attention share a common capacity limitation when conjunction search relies strongly on serial item selection.
\end{abstract}

Keywords Visual attention · Visual search $\cdot$ Central attention $\cdot$ Response selection $\cdot$ Locus-of-slack method

Capacity limitations are a main characteristic of the cognitive system. Often, different types of capacity limitations have been studied in isolation, but shedding light on their interplay is as important to unravel the architecture of human cognition. Here, the interplay of visual attention and central attention is in the focus of interest. In many situations, visual attention is necessary to select objects and bind object features because humans cannot attend to all objects in the visual field at the same time, showing that visual attention is limited in capacity (Treisman \& Gelade, 1980; Treisman \& Sato, 1990; Wolfe, 1994, 2007, 2012). In addition, central attention is assumed to be limited in capacity, as manifested by the difficulties that

Christina B. Reimer

christina.reimer@psych.uni-halle.de

1 Department of Psychology, Martin-Luther-Universität Halle-Wittenberg, Emil-Abderhalden-Str. 26-27, 06108 Halle (Saale), Germany

2 Department of Experimental Psychology, Ghent University, Henri Dunantlaan 2, 9000 Ghent, Belgium arise when humans perform two or more tasks simultaneously. Such performance decrements suggest that central processes like response selection can only be carried out for one task at a time (Pashler, 1994; Schubert, 1999; Welford, 1952; but see Meyer \& Kieras, 1997; Navon \& Miller, 2002; Tombu \& Jolicoeur, 2003, for alternative assumptions). The question arises whether visual and central attention influence each other, indicating that they share a capacity limitation, or whether they operate independently, indicating that they rely on distinct capacity limitations. Based on previous findings of our group (Reimer \& Schubert, 2019; Reimer, Strobach, Frensch, $\&$ Schubert, 2015), we further investigated this question in the present study in two dual-task experiments with a focus on the visual search demands.

In feature integration theory (FIT), Anne Treisman laid the groundwork for explaining the fundamental mechanisms of visual attention (Treisman, 1988, 1996, 1998, 2006; Treisman \& Gelade, 1980; Treisman \& Sato, 1990). In that sense, FIT can be considered as starting point for other theories on visual attention like guided search (Wolfe, 1994, 1998, 2007; Wolfe, Cave, \& Franzel, 1989) or similarity theory 
(Duncan \& Humphreys, 1989, 1992; Humphreys, Hodsoll, Olivers, \& Yoon, 2006; for reviews on visual attention, see Carrasco, 2011; Eckstein, 2011; Eimer, 2015). As is typical for studies on visual attention, visual search paradigms like feature search and conjunction search have been used to uncover the mechanisms of visual attention. In feature or pop-out search tasks, the target differs from the surrounding distractors in only one feature - for example, a red item among green items (Müller \& Krummenacher, 2006; Treisman \& Gelade, 1980; Wolfe, 1994, 1998, 2007). Target detection is fast, and adding distractors to the search display does not affect search times. Because search times are independent of set size (i.e., the number of items in the display), feature search is performed preattentively. In contrast, in regular conjunction search tasks, the target consists of a unique combination of two features and every distractor shares one of the target features - for example, a red vertical bar among red horizontal and green vertical bars. Visual attention is required to select the items and to bind the item features-for example, color and form - so that an attended item can be identified as target or distractor (Treisman, 1988, 1996, 1998, 2006; Treisman \& Gelade, 1980; Wolfe, 1994, 2007, 2012; Wolfe \& Bennett, 1997; but see Di Lollo, 2012). Because visual attention is capacity limited, it is assumed that feature binding cannot be done simultaneously for all items in the display, but rather the items are selectively attended, which results in a serial search process. Consequently, search time increases as distractors are added to the search display. The increase in search time with larger set size is called set size effect and reflects that visual attention is limited in capacity (Treisman \& Gelade, 1980; Wolfe, 1994, 2007; Wolfe et al., 1989).

The potential limitation of central attention has been studied with the psychological refractory period (PRP) paradigm (Pashler, 1994; Welford, 1952; for a review, see Fischer \& Plessow, 2015). In the PRP paradigm, two choice reaction time (RT) tasks, Task 1 and Task 2, are presented with variable temporal intervals (stimulus onset asynchronies [SOAs]). Both tasks require fast and accurate responses with priority on Task 1. As a typical finding, RT of Task 2 (i.e., RT2) decreases with longer SOA (i.e., PRP effect; Pashler, 1994; Schubert, 1999, 2008), whereas RT of Task 1 (i.e., RT1) is usually unaffected by the SOA manipulation (but see Strobach, Schütz, \& Schubert, 2015).

Competing theories account for the PRP effect. Following the central bottleneck model, each task can be roughly decomposed into perception, response selection, and motor response stages. In general, perception and motor response are independent of central attention and operate in parallel to the other processing stages. Response selection, however, requires central attention to bind perceived stimulus to associated response information. The model suggests that central attention is only available for one task at a time, which makes response selection a bottleneck stage (Pashler, 1994; Schubert,
1999, 2008; Sigman \& Dehaene, 2006; Welford, 1952; but see Huestegge \& Koch, 2010; Logan \& Gordon, 2001; Meyer \& Kieras, 1997; Navon \& Miller, 2002; Tombu \& Jolicoeur, 2003). Accordingly, at short SOA, response selection in Task 2 is processed only once central attention is available again, that is after response selection in Task 1 has been completed. The waiting time for response selection in Task 2, which is called slack, is prolonged and mirrored in increasing RT2.

However, the structural character of the central bottleneck model has been challenged. Meyer and Kieras (1997) proposed a strategic bottleneck model that includes the possibility of parallel response selection performance in dual-tasks. Others developed central capacity sharing models (Navon \& Miller, 2002; Tombu \& Jolicoeur, 2003). According to such resource models, a limited processing capacity can be flexibly allocated to the first and the second task in a dual-task. Sharing limited processing capacity between both tasks implies the possibility of parallel response selection performance, under the condition that response selection performance in each task slows down. Although these models propose different explanations for the PRP effect, they have in common that dual-task performance suffers from limited central attention.

In a previous study (Reimer et al., 2015, Experiment 1), we already investigated whether visual attention and central attention rely on the same or on distinct capacity limitations by applying the locus-of-slack method (Schweickert, 1978, 1980). In the dual-task, an auditory Task 1 required the discrimination between a high and a low tone, while a regular conjunction search Task 2 required the detection of the presence versus absence of the target, a red vertical bar among green vertical and red horizontal distractor bars in displays of 6,12 , and 18 items. Both tasks were presented at variable SOAs from 50 to $800 \mathrm{~ms}$. The visual search time (i.e., RT2) analysis revealed an underadditive interaction of SOA and set size, that is, the set size effect at SOA 50 (i.e., RT2 difference between set sizes 18 and 6) was significantly reduced compared with the set size effect at SOA 800 (see Fig. 1A). According to the logic of the locus-of-slack method, the observation of an underadditive interaction can be reconciled with the assumption that visual search processes in Task 2 are processed in parallel to the perception and response selection stages (i.e., the bottleneck stage) of Task 1 . Therefore, the additional amount of visual search time for the large set size compared with the small set size can be absorbed into slack at short SOA 50, but not at long SOA 800 (see also Pashler \& Johnston, 1989; Schubert, Fischer, \& Stelzel, 2008). The finding of parallel processing of regular conjunction search in Task 2 and response selection in Task 1 points to the fact that in general, visual attention and central attention rely on distinct capacity limitations. Note that in case visual and central attention had relied on a common capacity limitation, the locus-of-slack method would have predicted similar set size 
A - Central attention and visual attention rely on distinct capacity limitations: Response selection (RS) and conjunction search (CS) are processed in parallel

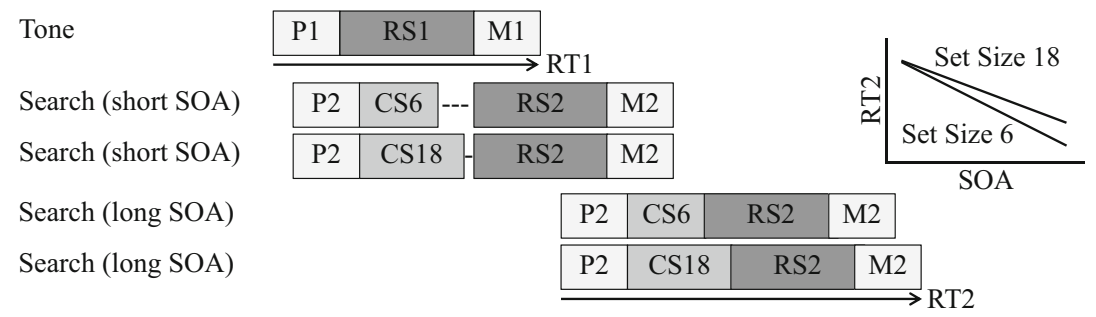

B - Central attention and visual attention rely on a common capacity limitation: Response selection (RS) and conjunction search (CS) are processed sequentially

Tone

Search (short SOA)

Search (short SOA)

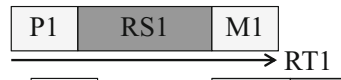

\begin{tabular}{|c|c|c|c|c|}
\hline P2 & -Slack- & CS6 & RS2 & M2 \\
\hline P2 & -Slack- & CS18 & RS2 & M2 \\
\hline
\end{tabular}

Search (long SOA)

\begin{tabular}{|c|c|c|c|}
\hline P2 & CS6 & RS2 & M2 \\
\hline \hline P2 & CS18 & RS2 & M2 \\
\hline
\end{tabular}

Search (long SOA)

Fig. 1 A If visual and central attention rely on distinct capacity limitations, an underadditive interaction of SOA and set size on reaction time of Task 2 (RT2) should result, indicating that conjunction search and response selection are performed concurrently at short SOA. The set size manipulation in Task 2 is shown for set sizes 6 and 18. B If visual and central attention share a common capacity limitation, additive effects of SOA and set size on RT2 should result, indicating that conjunction search

effects across SOAs, as would have been indicated by additive effects of SOA and set size (see Fig. 1B). Accordingly, visual search in Task 2 would have been performed only after response selection in Task 1 would have been finished (please see the General Discussion where we discuss a potential limitation of the locus-of-slack method).

In a recent study, Han (2017) also applied the locus-ofslack method in two dual-task experiments to examine the interplay of visual and central attention. In Experiment 2, the author used a conjunction search Task 2 with set sizes 3 and 6 . The target was a red, 45-degree tilted Gabor grating, and participants should indicate whether it was tilted to the right or to the left. Distractors were red vertical and green 45-degree tilted gratings. The underadditive interaction of SOA and set size showed that visual search time was absorbed into slack at short SOA compared with long SOA, which replicates the results of our study (Reimer et al., 2015, Experiment 1). However, most importantly, in Experiment 1, Han presented an orientation search Task 2 that requires more serial item selection than the regular conjunction search task. In the orientation search task with set sizes 3 and 6, all Gabor gratings were of the same color. The target was a 45-degree tilted grating and participants should indicate whether it was tilted to the right or to the left. Distractors were horizontal and vertical gratings. The visual search time analysis yielded additive effects of SOA and set size, indicating that response selection in Task 1 and visual search in Task 2 were performed is performed after response selection at short SOA. The set size manipulation in Task 2 is shown for set sizes 6 and 18. RT1 = reaction time to Task $1 ; \mathrm{P} 1=$ perception stage of Task $1 ; \mathrm{RS} 1=$ response selection stage of Task $1 ; \mathrm{M} 1=$ motor stage of Task $1 ; \mathrm{RT} 2=$ reaction time to Task $2 ; \mathrm{P} 2=$ perception stage of Task 2; CS 6/18 = conjunction search set size 6/18; $\mathrm{RS} 2=$ response selection stage of Task 2; M2 = motor stage of Task 2; $\mathrm{SOA}=$ stimulus onset asynchrony

sequentially. On the basis of these findings, Han concluded that by default, visual and central attention rely on distinct capacity limitations, but that they share a capacity limitation when the demands for serial item selection in the visual search task are increased. This would imply that the characteristics of visual search tasks are decisive for finding evidence in favor of different or shared visual and central attention limitations.

In the present study, we aimed at further investigating the role of visual search demands for the interplay of visual and central attention. As described above, Han (2017) demonstrated in an elegant way that depending on the visual search demands, central attention can be required for serial item selection in the visual search process. In Experiment 1, he used an orientation search task that in our view falls in the category of spatial configuration search. In our previous work, however, we used conjunction search tasks. Spatial configuration search and conjunction search may differ with respect to the seriality of the search process, as was shown for example by Wolfe, Palmer, and Horowitz (2010). We aimed at building on Han's findings by testing for a new set of conjunction search tasks whether visual and central attention share a common capacity limitation. It was important for us to apply conjunction search tasks again, as they are typically used to study the role of visual attention in serial item selection and feature binding, which is a cornerstone of Anne Treisman's work (Treisman, 1988, 1996, 1998, 2006). If we understand Han's work correctly, in Experiment 1, he was more concerned with 
the seriality of the search process instead of the role of feature binding. However, as mentioned above, we aimed at using search tasks that explicitly rely on feature binding while requiring a serial search process at the same time. For that purpose, we conducted two experiments in which we presented a so-called triple conjunction search task and a so-called plus conjunction search task as Tasks 2 . In both experiments, Task 1 required an auditory two-choice discrimination. In the triple conjunction search Task 2, each item consisted of three features - for example, color, form, and size (Wolfe et al., 1989, Experiment 9), and target and distractors shared two features. In the plus conjunction search Task 2, each item consisted of four features, for example two color and two form features (Treisman, 2006; Wolfe, 2012; Wolfe \& Bennett, 1997, Experiment 1), and target and distractors shared all four features. As will be explained in more detail below, although both search tasks were supposed to require serial item selection and feature binding, we were interested in investigating the involvement of central attention under the conditions of increased demands for serial item selection. The present study thus complements our previous work on the interplay of visual and central attention by extending it from conjunction search tasks of two features per item to conjunction search tasks of three and four features per item.

The rationale behind the choice of conjunction search tasks in Experiments 1 and 2 is as follows. To highlight the importance of Anne Treisman's work, we will focus on FIT to explain the expected performance differences in triple and plus conjunction search compared with regular conjunction search (Treisman, 1988, 1998, 2006; Treisman \& Gelade, 1980; Treisman \& Sato, 1990). FIT assumes that focused attention is required to bind item features in conjunction search. Crucially, the need for feature binding in a specific conjunction search task depends on the discriminability of the target compared with the distractors. In regular conjunction search, each item consists of two features, and target and distractors share one feature - for example, a red vertical target among red horizontal and green vertical distractors. FIT further assumes that the search process could be strategically adjusted so that the deployment of focused attention and feature binding could be restricted to the relevant group of distractors in an item-by-item manner until target detection, while the irrelevant group of distractors could be inhibited. That is, given a red target, it should be sufficient to deploy focused attention among the red distractors and bind their features, whereas the green distractors could be ignored (Treisman, 1998, 2006; Treisman \& Gelade, 1980). (Note that according to an alternative explanation on regular conjunction search performance, there should be the possibility that multiple conjunction search items share limited visual attentional capacity. In that case, conjunction search should be performed in a parallel manner. However, conjunction search performance would then slow down, resulting in set size effects; Sung, 2008; Townsend, 1971; see also Bichot, Rossi, \& Desimone, 2005; Huang \& Pashler, 2007; Thornton \& Gilden, 2007.)

In the triple conjunction search Task 2 in Experiment 1, each item consisted of a conjunction of three features. The target was big red vertical, and the three distractor groups consisted of big red horizontal, small red vertical, and big green vertical items (Wolfe et al., 1989, Experiment 9). According to FIT, the green distractor group would be irrelevant when searching for the red target and could be ignored. In turn, focused attention should be deployed among the two red distractor groups because they were the relevant distractors when searching for the red target (Treisman, 1998, 2006; see Fig. 2).

In Experiment 2, we used a plus conjunction search Task 2 in which target and distractors shared the same four features, so that the amount of feature overlap was maximal (Treisman, 1998, 2006; Wolfe, 2012; Wolfe \& Bennett, 1997, Experiment 1). The target was a plus with crossed red vertical and green horizontal bars, whereas distractors were plusses with crossed green vertical and red horizontal bars. Following FIT, in contrast to regular and triple conjunction search, it should be impossible to ignore any distractors in plus conjunction search. Instead, the search process should completely rely on serial item selection and feature binding to detect the target (see Fig. 2). In that sense, Experiment 2 can be considered as an attempt to replicate Han's findings (Han, 2017, Experiment 1).

To summarize, regular, triple, and plus conjunction searches differ with respect to the need for serial deployment of focused attention and feature binding that is closely linked to it. Following FIT, the fewer distractors could be ignored, the more serial shifts of focused attention should be necessary to detect the target. In the present study, we aimed at shedding more light on the role of central attention for serial shifts of focused attention. Accordingly, we hypothesized that central attention should be required in plus conjunction search, because in this search task, the need for serial attention shifts should be maximal, as none of the distractors could be ignored. That is why we expected additive effects of SOA and set size for this search task (see Fig. 1B). However, different outcomes were possible with regard to the question of whether or not central attention would also be required for serial shifts of focused attention in triple conjunction search, as one group of distractors could still be ignored in this search task. While additive effects of SOA and set size would point to the role of central attention in triple conjunction search (see Fig. 1B), an underadditive interaction of SOA and set size would provide evidence against it (see Fig. 1A).

\section{Experiment 1}

In Experiment 1, participants were presented with an auditory two-choice discrimination Task 1 and a triple conjunction 


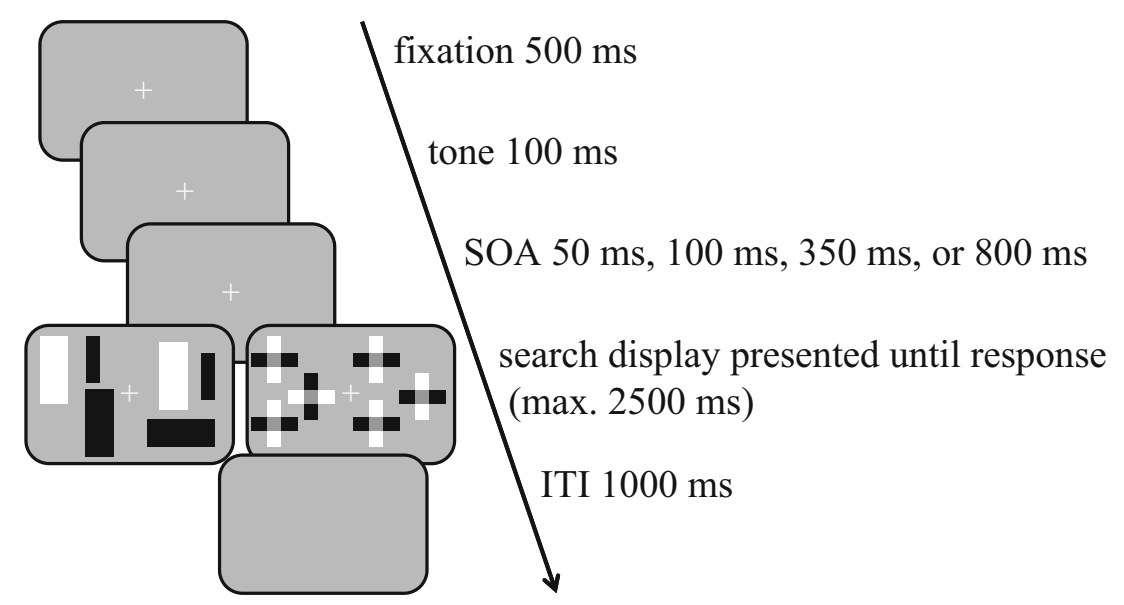

Fig. 2 An example trial in Experiments 1 and 2 that consisted of auditory two-choice discrimination Tasks 1 and a triple conjunction search Task 2 (Experiment 1) or a plus conjunction search Task 2 (Experiment 2). Here, the condition target present set size 6 is presented. Originally, in Experiment 1, there were one big red vertical target (shown in black), two small red vertical distractors (shown in black), one big red horizontal distractor (shown in black), and two big green vertical distractors (shown in white). Originally, in Experiment 2, the target was a plus made of a small red vertical (shown in black) and a small green horizontal bar (shown in white), whereas the distractors were plusses made of small green vertical (shown in white) and small red horizontal bars (shown in black). $\mathrm{ms}=$ millisecond; $\mathrm{SOA}=$ stimulus onset asynchrony; ITI = intertrial interval search Task 2. In the search task, participants were asked to report the presence versus absence of a big red vertical target among small red vertical, big red horizontal, and big green vertical distractors (Wolfe et al., 1989, Experiment 9). If the search process based on serial item selection and feature binding is independent of central attention, there should be an underadditive interaction of SOA and set size (see Fig. 1A). This finding would be similar to the results based on a regular conjunction search Task 2 (Reimer \& Schubert, 2019; Reimer et al., 2015; Wolfe et al., 1989, Experiment 9) and would provide more evidence that visual and central attention rely on distinct capacity limitations. However, if the search process requires central attention for serial item selection and feature binding, there should be additive effects of SOA and set size (see Fig. 1B). This finding would indicate that visual and central attention share a common capacity limitation (see Han, 2017, Experiment 1).

\section{Method}

Participants In Experiment 1, there were 24 participants (five men, 19 women, all right-handed, all normal or corrected-tonormal vision) with a mean age of 23.1 years $(S D=4.9$ years, age range: $18-35$ years). The sample size was chosen based on the sample sizes of our previous experiments on this topic (Reimer \& Schubert, 2019; Reimer et al., 2015). The participants were psychology students of the Martin-LutherUniversität Halle-Wittenberg and received course credit for their participation. Written informed consent was obtained from all participants before the experiment.
Apparatus and stimuli The experiment was programmed in Presentation. The stimuli of the conjunction search Task 2 were presented on a 24-inch LED-TFT monitor at a resolution of $1,920 \times 1,080$ pixels with a refreshing rate of $100 \mathrm{~Hz}$ at a viewing distance of $60 \mathrm{~cm}$. We adapted the triple conjunction search task from a study of Wolfe et al. (1989, Experiment 9). Participants were asked to indicate the presence versus absence of a big red vertical bar, the target, among three different distractor types: small red vertical, big red horizontal, and big green vertical bars (red: CIE: $x=0.620, y=0.346$; luminance $=$ $65.22 \mathrm{~cd} / \mathrm{m}^{2}$; green: CIE: $x=0.342, y=0.566$; luminance $=$ $\left.100.80 \mathrm{~cd} / \mathrm{m}^{2}\right)$. The stimuli were presented on a dark background (CIE: $x=0.313, y=0.329$; luminance $=12.46 \mathrm{~cd} /$ $\mathrm{m}^{2}$ ). The distribution of distractors for set sizes 6,12 , and 18 for target present trials was as follows: two, four, and six small red vertical distractors for set sizes 6,12 , and 18 ; one, three, and five big red horizontal distractors for set sizes 6,12 , and 18 , and two, four, and six big green vertical distractors for set sizes 6 , 12 , and 18 . For target absent trials, the distractors were evenly distributed for set sizes 6,12, and 18-that is, two of each distractor for set size 6 , four of each for set size 12 , and six of each for set size 18 . On every trial, the items were randomly presented on six, 12 or 18 of 32 positions of an invisible grid with a size of $100 \times 80 \mathrm{~mm}$. Items were never presented on the vertical and horizontal midlines. The small items had a size of 6 $\times 2 \mathrm{~mm}$, and the big items of $9 \times 3 \mathrm{~mm}$. The white central fixation point had a size of $1 \times 1 \mathrm{~mm}$.

Task 1 of the dual-task consisted of an auditory two-choice discrimination task. Participants should discriminate between two sine-wave tones of $350 \mathrm{~Hz}(78 \mathrm{~dB})$ and $900 \mathrm{~Hz}(80 \mathrm{~dB})$. The tones were presented for $100 \mathrm{~ms}$ via headphones. 
Four keys of a QWERTZ keyboard served as response keys. Participants were asked to respond to the low and the high tones by pressing the " $\mathrm{Y}$ " and the " $\mathrm{X}$ " keys with the middle and index fingers of their left hand, respectively. They were asked to respond to target present and target absent by pressing the comma (,) and the period (.) keys with the index and middle fingers of their right hand, respectively. Each of the four keys but none of the other keys of the keyboard could be used to start a new block after a break.

Design and procedure We used a within-subjects design that consisted of orthogonally combining four SOAs $(50 \mathrm{~ms}$ vs. $100 \mathrm{~ms}$ vs. $350 \mathrm{~ms}$ vs. $800 \mathrm{~ms}) \times 3$ set sizes $(6$ vs. 12 vs. 18$) \times$ 2 display types (target present vs. target absent). The 24 combinations were presented twice per block with the low tone in Task 1 and twice per block with the high tone in Task 1 in random order, which resulted in 96 trials per block. There were eight blocks in each experiment; thus, every combination was presented 16 times.

Before completing the experimental blocks, participants were asked to complete 48 practice trials of the auditory Task 1 ( 24 practice trials for the low tone and for the high tone, respectively) followed by 48 practice trials of the conjunction search Task 2 (24 practice trials for target present and for target absent, respectively). Afterwards, participants practiced both tasks in a dual-task block consisting of 48 trials. As in the experimental blocks, they were asked to respond as quickly and as accurately as possible to both tasks with priority on Task 1.

As shown in Fig. 2, each trial started with the fixation point shown at the center of the screen. After $500 \mathrm{~ms}$ of fixation, one of the two tones was played for $100 \mathrm{~ms}$. Following the SOA of either $50 \mathrm{~ms}, 100 \mathrm{~ms}, 350 \mathrm{~ms}$, or $800 \mathrm{~ms}$, the search display was presented until participants responded, but the maximum duration was $2,500 \mathrm{~ms}$. There was an intertrial interval (ITI) of $1,000 \mathrm{~ms}$ showing a blank dark screen after the response to Task 2.

\section{Results}

RTs of Task 2 and Task 1 were separately analyzed with repeated measures analysis of variance (ANOVAs) with the three factors SOA, set size, and display type. ${ }^{1}$ Because we were interested in the interplay of central attention in Task 1 and visual attention in Task 2, we could only derive valid conclusions when both tasks were performed correctly in a trial. Therefore, in the RT analysis, $9.67 \%$ of trials with errors in Task 1 and/or Task 2 were excluded (Task 1: $2.04 \%$ and Task 2: $7.66 \%$ ). Error trials included incorrectly committed and omitted responses to Task 1 and/or Task 2. Error rates of Task 2 and Task 1 were transformed according to the formula

\footnotetext{
${ }^{1}$ Data and materials are available upon request from the authors.
}

$2 \sin ^{-1} \sqrt{\text { error rate }}$ (Kirk, 2013) before they were submitted to the same ANOVAs as RTs. Error rates were arc-sine transformed so that the distribution of the proportional data was more likely to be symmetrical and in turn the assumption of normality was more likely to be met before running the same repeated-measures ANOVAs as for RT. When assumptions of sphericity were violated, $p$ values were adjusted using the Greenhouse-Geisser correction.

RT triple conjunction search Task 2 RT2 is presented separately for target present and target absent in Fig. 3A and B. There was a PRP effect of $302 \mathrm{~ms}$. RT2 decreased from 1,223 ms at SOA 50 to $921 \mathrm{~ms}$ at $\mathrm{SOA} 800, F(3,69)=157.486, p<.001, \eta_{\mathrm{p}}{ }^{2}=.873$. Moreover, RT2 increased from $950 \mathrm{~ms}$ for set size 6 to $1,204 \mathrm{~ms}$ for set size 18, showing a set size effect of $254 \mathrm{~ms}, F(2,46)=$ $232.178, p<.001, \eta_{\mathrm{p}}{ }^{2}=.910$. RT2 was $144 \mathrm{~ms}$ faster for target present $(M=1,008 \mathrm{~ms})$ compared with target absent $(M=1,152$ $\mathrm{ms}), F(1,23)=149.975, p<.001, \eta_{\mathrm{p}}{ }^{2}=.867$. The interaction of set size and display type revealed a larger set size effect for target absent $(M=308 \mathrm{~ms})$ compared with target present $(M=202 \mathrm{~ms})$, $F(2,46)=24.621, p<.001, \eta_{\mathrm{p}}{ }^{2}=.517$. For target present, RT2 increased from $902 \mathrm{~ms}$ for set size 6 to $1,104 \mathrm{~ms}$ for set size 18 . For target absent, RT2 increased from $997 \mathrm{~ms}$ for set size 6 to $1,305 \mathrm{~ms}$ for set size 18 .

The interaction of SOA and set size was significant, $F(6$, $138)=5.870, p<.001, \eta_{\mathrm{p}}{ }^{2}=.203$. The set size effect at SOA $50(M=215 \mathrm{~ms})$ was significantly smaller compared with the set size effect at SOA $800(M=295 \mathrm{~ms}), t(23)=3.832, p<$ .001 . Eighty $\mathrm{ms}$ of visual search time were absorbed into slack. Other interactions were not significant.

The search slopes shown in Table 1 increased from 17.89 $\mathrm{ms} /$ item at SOA 50 to $24.55 \mathrm{~ms} /$ item at SOA $800, F(3,69)=$ $10.769, p<.001, \eta_{\mathrm{p}}{ }^{2}=.319$. Moreover, slopes differed between target absent $(M=25.62 \mathrm{~ms} /$ item $)$ and target present $(M$ $=16.81 \mathrm{~ms} /$ item $), F(1,23)=33.112, p<.001, \eta_{\mathrm{p}}{ }^{2}=.590$. The difference between target absent and target present was smallest at SOA 50 (absent vs. present: $M=19.87 \mathrm{~ms} /$ item vs. $15.84 \mathrm{~ms} /$ item), and larger at the other SOAs (e.g., SOA 800 , absent vs. present: $M=29.76 \mathrm{~ms} /$ item vs. $19.35 \mathrm{~ms} /$ item), $F(3,69)=2.722, p<.05, \eta_{\mathrm{p}}^{2}=.106$.

RT tone Task 1 RT1 is presented separately for target present and target absent in Fig. 3C and D. RT1 decreased from $839 \mathrm{~ms}$ at SOA 50 to $728 \mathrm{~ms}$ at SOA $800, F(3,69)=13.618, p<.001, \eta_{\mathrm{p}}{ }^{2}=$ .372. In addition, RT1 increased from $753 \mathrm{~ms}$ for set size 6 to $811 \mathrm{~ms}$ for set size $18, F(2,46)=14.994, p<.001, \eta_{\mathrm{p}}{ }^{2}=.395$. The factors SOA and set size interacted significantly, $F(6,138)=$ $3.152, p<.001, \eta_{\mathrm{p}}{ }^{2}=.121$, pointing to smaller set size effects with longer SOAs. That is, at SOA 50, RT1 increased from $797 \mathrm{~ms}$ for set size 6 to $877 \mathrm{~ms}$ for set size 18. At SOA 800, however, the increase was from $719 \mathrm{~ms}$ for set size 6 to $739 \mathrm{~ms}$ for set size 18. Other main effects or interactions were not significant. 


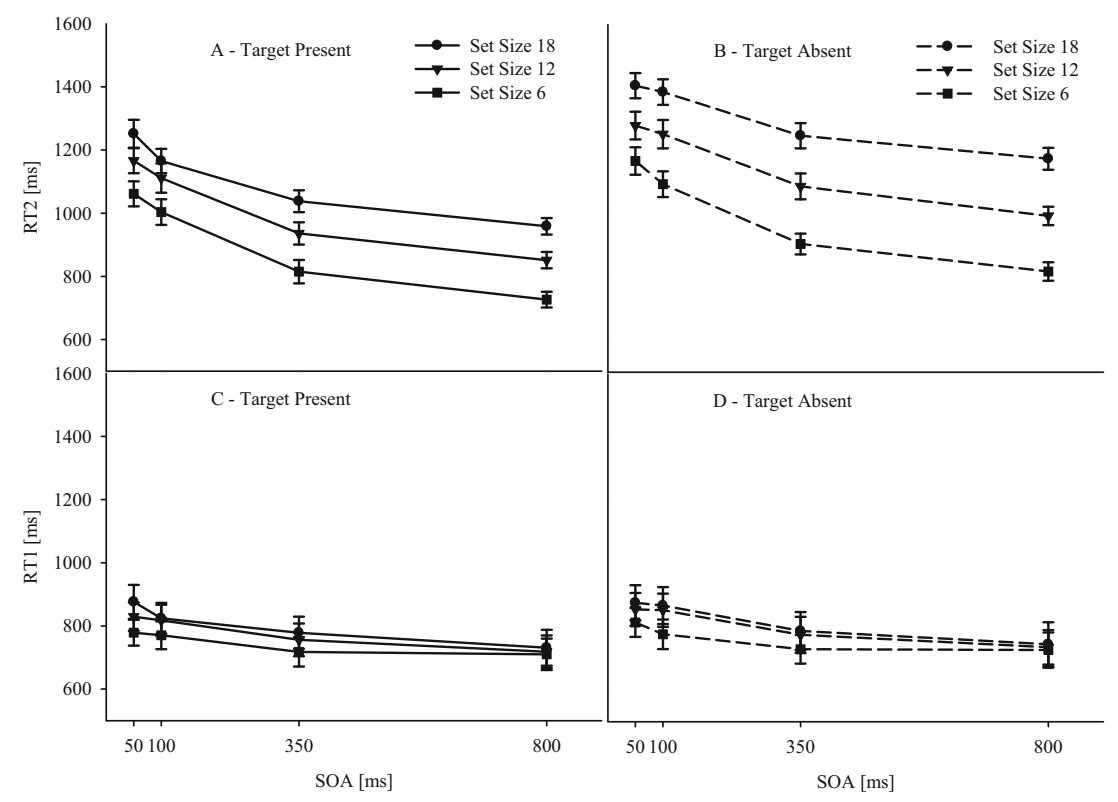

Fig. 3 Experiment 1: Mean reaction time for the triple conjunction search Task 2 (RT2) and the auditory two-choice discrimination Task 1 (RT1) as a function of stimulus onset asynchrony (SOA) and set size $(6,12$, and

\section{Error rates triple conjunction search Task 2 and tone Task 1} The error rates in percentages are presented in Table 2. In triple conjunction search Task 2, error rates increased with increasing set size from $5.16 \%$ for set size 6 to $10.58 \%$ for set size $18, F(2,46)=43.462, p<.001, \eta_{\mathrm{p}}{ }^{2}=.654$. Error rates were higher for target present $(M=10.47 \%)$ compared with target absent $(M=4.84 \%), F(1,23)=111.490, p<.001, \eta_{\mathrm{p}}{ }^{2}=$ .829. The factors set size and display type interacted, $F(2,46)$ $=30.383, p<.001, \eta_{\mathrm{p}}{ }^{2}=.569$. Error rates were similar across set sizes 6,12 , and 18 for target absent $(M=4.39 \%, 4.56 \%$, and $5.57 \%$ ), but for target present, error rates increased with increasing set size $(M=5.92 \%, 9.90 \%$, and $15.59 \%)$. Moreover, error rates were higher for target present compared with target absent across SOAs (target present vs. absent, SOA 50 : $M=10.98 \%$ vs. $5.60 \%$; SOA $100: M=9.73 \%$ vs. $5.08 \%$; SOA $800: M=9.77 \%$ vs. $4.60 \%$ ) especially at SOA $350(M=11.41 \%$ vs. $4.08 \%), F(3,69)=3.217, p<.05, \eta_{\mathrm{p}}{ }^{2}=$ .123 .

Table 1 Mean search slopes ( $\mathrm{ms} /$ item) for the triple conjunction search Task 2 in Experiment 1 and the plus conjunction search Task 2 in Experiment 2 as a function of SOA and display type, respectively

\begin{tabular}{|c|c|c|c|c|c|c|c|c|}
\hline \multirow[b]{3}{*}{ Experiment } & \multicolumn{4}{|c|}{ Target absent } & \multicolumn{4}{|c|}{ Target present } \\
\hline & \multicolumn{4}{|c|}{ SOA (ms) } & \multicolumn{4}{|c|}{ SOA (ms) } \\
\hline & 50 & 100 & 350 & 800 & 50 & 100 & 350 & 800 \\
\hline 1 & 19.88 & 24.31 & 28.55 & 29.76 & 15.84 & 13.47 & 18.58 & 19.35 \\
\hline 2 & 26.11 & 26.79 & 30.04 & 27.77 & 18.08 & 17.32 & 18.98 & 20.32 \\
\hline
\end{tabular}

Note. $\mathrm{SOA}=$ stimulus onset asynchrony; $\mathrm{ms}=$ millisecond
18). A and B: RT2 graphs for target present and target absent. C and D: RT1 graphs for target present and target absent in the triple conjunction search Task 2. Error bars represent standard error of the mean

In the auditory Task 1 , error rates were higher at SOAs 50 $(M=2.58 \%)$ and $100(M=2.63 \%)$ compared with SOAs 350 $(M=1.45 \%)$ and $800(M=1.50 \%), F(3,69)=7.215, p<.001$, $\eta_{\mathrm{p}}{ }^{2}=.239$. Other effects or interactions were not significant.

\section{Discussion}

In Experiment 1, we investigated whether central attention was required for serial item selection and feature binding in triple conjunction search Task 2 . Eighty ms of visual search time were absorbed into slack, and the interaction of SOA and set size was significant. According to the locus-of-slack method, the result shows that a part of triple conjunction search in Task 2 was performed in parallel to response selection in Task 1.

Although $80 \mathrm{~ms}$ of visual search time were absorbed into slack, the set size effects across SOAs were still fairly large (see Fig. 3A-B). The large set size effects suggested that serial shifts of visual attention were required to detect the target in triple conjunction search. An analysis of performance in the auditory Task 1 could help to reconcile these seemingly contradictory observations. In more detail, for RT1, the interaction of SOA and set size was also significant. At SOA 50, there was a set size effect of $80 \mathrm{~ms}$. Slower Task 1 performance at short SOA is in general associated with a longer slack time, which allows for more visual search time to be absorbed into slack (Schweickert, 1978, 1980). Because the set size effect at short SOA corresponded to the amount of visual search time that was absorbed into slack, it is possible that participants performed response selection in Task 1 and a part of triple conjunction search in Task 2 in parallel solely by slowing down response selection in Task 1 . In other words, it 
Table 2 Mean error rates (in \%) for the triple conjunction search Task 2 and the auditory two-choice discrimination Task 1 in Experiment 1 as well as for the plus conjunction search Task 2 and the auditory two-choice discrimination Task 1 in Experiment 2 as a function of SOA and Task 2 manipulation (set size, display type), respectively

\begin{tabular}{|c|c|c|c|c|c|c|c|c|c|c|}
\hline \multirow[b]{3}{*}{ Task } & \multirow[b]{3}{*}{ Set size } & \multirow[b]{3}{*}{ Display type } & \multicolumn{4}{|c|}{ Experiment 1} & \multicolumn{4}{|c|}{ Experiment 2} \\
\hline & & & \multicolumn{4}{|c|}{ SOA (ms) } & \multicolumn{4}{|c|}{ SOA (ms) } \\
\hline & & & 50 & 100 & 350 & 800 & 50 & 100 & 350 & 800 \\
\hline \multirow[t]{6}{*}{2} & \multirow[t]{2}{*}{6} & Absent & 5.47 & 4.56 & 4.17 & 3.39 & 5.73 & 5.21 & 4.43 & 3.26 \\
\hline & & Present & 6.12 & 4.95 & 6.77 & 5.86 & 12.50 & 11.33 & 11.33 & 13.80 \\
\hline & \multirow[t]{2}{*}{12} & Absent & 5.34 & 5.08 & 3.13 & 4.69 & 10.16 & 8.85 & 7.55 & 6.64 \\
\hline & & Present & 9.09 & 10.81 & 9.64 & 9.24 & 21.88 & 21.22 & 22.27 & 25.13 \\
\hline & \multirow[t]{2}{*}{18} & Absent & 5.99 & 5.60 & 4.95 & 5.73 & 16.28 & 14.19 & 9.64 & 8.20 \\
\hline & & Present & 16.93 & 13.41 & 17.84 & 14.19 & 30.08 & 31.12 & 30.99 & 31.90 \\
\hline \multirow[t]{6}{*}{1} & \multirow[t]{2}{*}{6} & Absent & 2.73 & 3.78 & 1.82 & 1.30 & 3.52 & 2.99 & 2.47 & 1.56 \\
\hline & & Present & 2.47 & 1.82 & 1.43 & 2.60 & 4.04 & 1.95 & 2.21 & 1.69 \\
\hline & \multirow[t]{2}{*}{12} & Absent & 3.13 & 2.60 & 1.43 & 1.56 & 2.08 & 2.08 & 1.30 & 1.43 \\
\hline & & Present & 2.21 & 2.34 & 1.70 & 1.17 & 3.52 & 2.21 & 1.69 & 1.30 \\
\hline & \multirow[t]{2}{*}{18} & Absent & 2.21 & 2.47 & 1.43 & 1.30 & 2.34 & 2.47 & 1.95 & 1.82 \\
\hline & & Present & 2.73 & 2.73 & 0.91 & 1.04 & 3.13 & 1.95 & 2.60 & 0.78 \\
\hline
\end{tabular}

Note. $\mathrm{SOA}=$ stimulus onset asynchrony; $\mathrm{ms}=$ millisecond

might be the case that if RT1 had not been slowed down at short SOA, a part of triple conjunction search in Task 2 would probably not have been performed during slack, which would have resulted in additive effects of SOA and set size. Such a finding would have presented clearer evidence for the assumption that central attention is required for serial shifts of visual attention in triple conjunction search Task 2.

In Experiment 2, we tested the role of central attention in plus conjunction search. Both triple and plus conjunction searches require serial shifts of visual attention and feature binding to detect the target. However, in theory, one group of distractors could still be ignored in triple, but not in plus conjunction search, which could lead to a different requirement of central attention in plus conjunction search (Treisman, 1998, 2006).

\section{Experiment 2}

In Experiment 2, participants were presented with the same auditory two-choice discrimination Task 1 , but with a plus conjunction search Task 2, where feature overlap between target and distractors was maximal (Treisman, 1998, 2006; Wolfe, 2012; Wolfe \& Bennett, 1997, Experiment 1). Participants were asked to report the presence versus absence of the target, a plus with crossed red vertical and green horizontal bars, whereas all distractors were plusses with crossed green vertical and red horizontal bars. Because target and distractors shared the same four features, it was not possible to ignore distractors. Rather, an item had to be selected before it was identified as target or distractor, which was accomplished in a serial search process (Treisman, 1998, 2006; Wolfe, 2012; Wolfe \& Bennett, 1997, Experiment 1). Consequently, we expected additive effects of SOA and set size indicating that plus conjunction search in Task 2 would be processed after response selection in Task 1 has been completed. Accordingly, this finding would provide evidence that visual and central attention share a common capacity limitation, when central attention is involved in selecting the items in a serial search process (see Fig. 1B). Moreover, Experiment 2 could be considered as attempt to replicate Han's (2017, Experiment 1) result based on an orientation search task with a conjunction search task.

\section{Methods}

In Experiment 2, there were 24 new participants (four men, 20 women, all right-handed, all normal or corrected-to-normal vision) with a mean age of 24.4 years $(S D=4.1$ years, age range: 18-37 years). Four were psychology students of the Martin-Luther-Universität Halle-Wittenberg and 20 were psychology students of the Humboldt-Universität zu Berlin. All of them received course credit for their participation. Written informed consent was obtained from all participants before the experiment.

Experiment 2 was similar to Experiment 1, except that a plus conjunction search Task 2, as shown in Fig. 2, was used (Treisman, 1998, 2006; Wolfe, 2012; Wolfe \& Bennett, 1997, Experiment 1). The target plus consisted of a small red vertical and a small green horizontal bar, whereas the bars were 
switched for the distractors. A black square was inserted in the middle of each plus to prevent foreground-background segmentation of the two bars.

\section{Results}

The data analysis was the same as in Experiment 1. In Experiment 2, $17.37 \%$ of error trials were excluded (Task 1: $2.21 \%$ and Task 2: $15.15 \%$ ).

RT plus conjunction search Task 2 RT2 is presented separately for target present and target absent in Fig. 4A and B. RT2 decreased from $1,539 \mathrm{~ms}$ at SOA 50 to $1,306 \mathrm{~ms}$ at SOA 800 , revealing a PRP effect of $233 \mathrm{~ms}, F(3,69)=94.153, p$ $<.001, \eta_{\mathrm{p}}{ }^{2}=.804$. There was a set size effect of $278 \mathrm{~ms}$ - that is, RT2 increased from $1281 \mathrm{~ms}$ for set size 6 to $1559 \mathrm{~ms}$ for set size $18, F(2,46)=160.797, p<.001, \eta_{\mathrm{p}}{ }^{2}=.875$. RT2 was $353 \mathrm{~ms}$ slower for target absent $(M=1,613 \mathrm{~ms})$ compared with target present $(M=1,260 \mathrm{~ms}), F(1,23)=87.443, p<$ $.001, \eta_{\mathrm{p}}{ }^{2}=.792$. The interaction of set size and display type revealed a larger set size effect for target absent $(M=333 \mathrm{~ms})$ compared with target present $(M=224 \mathrm{~ms}), F(2,46)=25.995$, $p<.001, \eta_{\mathrm{p}}{ }^{2}=.531$. For target present, RT2 increased from $1,142 \mathrm{~ms}$ for set size 6 to $1,366 \mathrm{~ms}$ for set size 18 . For target absent, RT2 increased from 1,419 ms for set size 6 to $1752 \mathrm{~ms}$ for set size 18 .

The factors SOA and set size did not interact, $F(6,138)=$ $1.461, p=.196, \eta_{\mathrm{p}}{ }^{2}=.060$. Set size effects (i.e., RT2 difference between set sizes 6 and 18) across SOAs 50, 100, 350, and 800 were $265 \mathrm{~ms}, 264 \mathrm{~ms}, 294 \mathrm{~ms}$, and $289 \mathrm{~ms}$, respectively. None of the other interactions was significant.
The search slopes shown in Table 1 differed between target absent $(M=27.68 \mathrm{~ms} /$ item $)$ and target present $(M=18.67 \mathrm{~ms} /$ item), $F(1,23)=23.001, p<.001, \eta_{\mathrm{p}}{ }^{2}=.500$. Other effects or interactions were not significant.

Based on the suggestion of an anonymous reviewer, we conducted a between-experiment comparison to test whether the interaction of the factors SOA and set size differed between triple conjunction search Task 2 (Experiment 1) and plus conjunction search Task 2 (Experiment 2). The interaction of the between-subject factor experiment and the withinsubject factors SOA and set size was not significant, $F(6,276)$ $=1.744, p=.125, \eta_{\mathrm{p}}{ }^{2}=.0 .37$.

RT tone Task 1 RT1 is presented separately for target present and target absent in Fig. 4C and D. RT1 decreased from $841 \mathrm{~ms}$ at SOA 50 to $767 \mathrm{~ms}$ at SOA $800, F(3,69)=4.085, p<.05, \eta_{\mathrm{p}}{ }^{2}$ $=.151$. In addition, RT1 increased from $797 \mathrm{~ms}$ for set size 6 to $816 \mathrm{~ms}$ for set size $18, F(2,46)=3.408, p<.05, \eta_{\mathrm{p}}{ }^{2}=.129$. Other effects or interactions were not significant.

\section{Error rates plus conjunction search Task 2 and tone Task 1} The error rates in percentages are presented in Table 2. In Task 2 , error rates increased with increasing set size from $8.45 \%$ for set size 6 to $21.55 \%$ for set size $18, F(2,46)=59.711, p<$ $.001, \eta_{\mathrm{p}}{ }^{2}=.722$. Error rates were higher for target present $(M$ $=21.96 \%)$ compared with target absent $(M=8.34 \%), F(1,23)$ $=25.429, p<.001, \eta_{\mathrm{p}}{ }^{2}=.525$. Set size and display type interacted, $F(2,46)=11.274, p<.01, \eta_{\mathrm{p}}{ }^{2}=.329$, showing that error rates increased with increasing set size, but more for target present compared with target absent (set size 6, target present vs. absent, $M=12.24 \%$ vs. $4.65 \%$; set size 12 , target

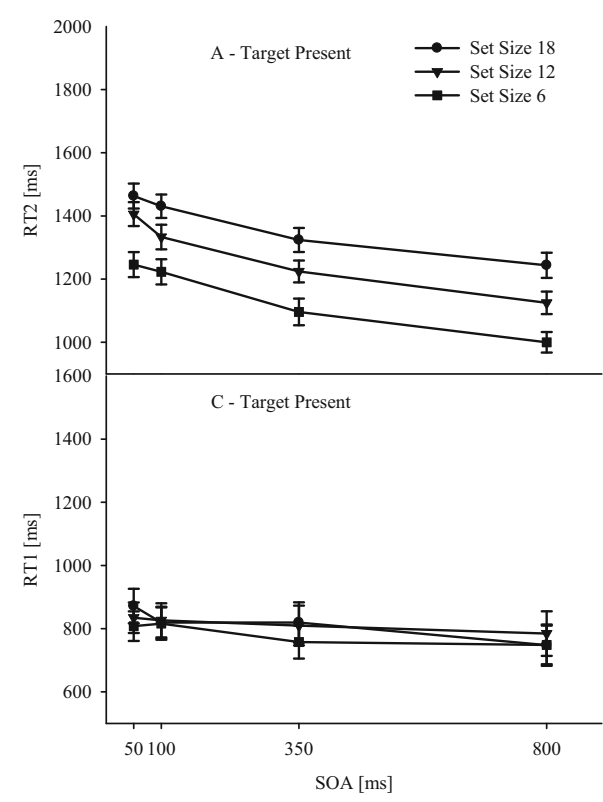

Fig. 4 Experiment 2: Mean reaction time for the plus conjunction search Task 2 (RT2) and the auditory two-choice discrimination Task 1 (RT1) as a function of stimulus onset asynchrony (SOA) and set size $(6,12$, and

18). A and B: RT2 graphs for target present and target absent. C and D: RT1 graphs for target present and target absent in the plus conjunction search Task 2. Error bars represent standard error of the mean 
present vs. absent, $M=22.62 \%$ vs. $8.30 \%$; set size 18 , target present vs. absent, $M=31.02 \%$ vs. $12.08 \%$ ). Moreover, error rates for target present were higher compared with error rates for target absent across SOAs and increased from SOA 50 to SOA $800(M=21.48 \%$ vs. $23.61 \%)$, but error rates for target absent decreased from SOA 50 to SOA $800(M=10.72 \%$ vs. $6.03 \%), F(3,69)=9.890, p<.001, \eta_{\mathrm{p}}{ }^{2}=.301$. Other effects or interactions were not significant.

In Task 1, error rates were higher at SOAs 50 and $100(M=$ $3.13 \%$ and $2.28 \%)$ compared with SOAs 350 and $800(M=$ $2.04 \%$ and $1.43 \%), F(3,69)=5.320, p<.01, \eta_{\mathrm{p}}{ }^{2}=.188$. Other effects or interactions were not significant.

\section{Discussion}

Based on the additive effects of SOA and set size, we concluded that plus conjunction search in Task 2 and response selection of an auditory two-choice discrimination Task 1 were processed sequentially. The results indicated that visual and central attention share a common capacity limitation when the search process relied strongly on serial item selection and feature binding to detect the target (Treisman, 1998, 2006; Wolfe, 2012; Wolfe \& Bennett, 1997, Experiment 1). As such, Experiment 2 replicated Han's (2017, Experiment 1) findings.

In Experiment 2, RT1 was $74 \mathrm{~ms}$ slower at SOA 50 compared with SOA 800. As in Experiment 1, slower RT1 at short SOA could indicate that participants tried to perform response selection in Task 1 and visual search in Task 2 in parallel by delaying response selection in Task 1 . However, this strategy was not successful in Experiment 2, because there was no interaction of SOA and set size in RT1 and visual search time was barely absorbed into slack.

A puzzling finding concerns the between-experiment comparison, which showed that the factors SOA and set size did not significantly interact and that this was not different across the two experiments. Considering that the set size effects were fairly large in both triple and plus conjunction search Tasks 2 (at SOA 800, the set size effects were $295 \mathrm{~ms}$ for triple and $289 \mathrm{~ms}$ for plus conjunction search), the finding of the between-experiment comparison seems to show that both triple and plus conjunction search Tasks 2 required high demands for serial item selection and modulated the interplay of visual and central attention to a similar extent. Nevertheless, one should be cautious with that interpretation, because the separate analysis of Experiment 1 provided a significant interaction of SOA and set size on RT2, which would be consistent with the assumption that at least a part of the search time was absorbed into slack in that experiment. In this respect, the combined findings can be reconciled with the assumption that visual search processes can be processed sequentially to the response selection in Task 1 if visual search relies strongly on serial item selection as it is the case in conditions like the plus conjunction search Task 2.

\section{General discussion}

In two experiments, we investigated whether visual search demands modulate the interplay of visual and central attention. In Experiment 1, a triple conjunction search Task 2 was paired with an auditory two-choice discrimination Task 1. Visual search time was partially absorbed into slack, as indicated by the underadditive interaction of SOA and set size. However, the set size effects across SOAs were rather large suggesting that triple conjunction search Task 2 relied on a serial search process. Moreover, the set size effect for RT1 at short SOA corresponded to the amount of triple conjunction search time that was absorbed into slack. Therefore, it is possible that triple conjunction search in Task 2 would have been performed completely after response selection in Task 1 , if Task 1 had not been delayed. It remains open whether or not this might have provided clearer evidence that central attention is required for serial item selection and feature binding in triple conjunction search.

In Experiment 2, a plus conjunction search Task 2 was paired with an auditory two-choice discrimination Task 1. There were additive effects of SOA and set size, which indicated that plus conjunction search in Task 2 was performed after response selection in Task 1 . The search task relied strongly on a serial search process because target and distractors shared the same four features, and to identify an item as target or distractor it had to be selected. Accordingly, in situations that strongly require a serial search process, visual search in Task 2 and response selection in Task 1 are performed sequentially, revealing that central attention is required for serially selecting the items before visual attention binds their features.

Comparing the interactions of the factors SOA and set size between both experiments provided some evidence that triple and plus conjunction search Tasks 2 represented conditions under which response selection and visual search processes are scheduled in sequential order in dual-task situations. In particular, this is based on the view that the observed underadditive interaction of SOA and set size showed that at least a part of the search time in the triple conjunction search Task 2 of Experiment 1 could be absorbed into slack under certain conditions of dual-task processing - that is, if Task 1 response selection processing is delayed. In that respect, these findings allowed for the conclusion that visual search demands modulated the interplay of visual and central attention insofar as that visual and central attention share a common capacity limitation when the visual search task relies heavily on serial item selection.

One of the reviewers pointed to an implicit assumption of the locus-of-slack method. To reveal whether the process of interest in Task 2 (here, visual search) is performed in parallel or sequentially to response selection in Task 1 , the duration of that process should not exceed the duration of slack. The 
reviewer was wondering what happens if the visual search process cannot be completed during slack. This is indeed an interesting question. In the present study, the duration of slack could be approximated by the PRP effect. Comparing the PRP effect to the set size effect at SOA 800 could provide a first estimate of whether it is actually possible that visual search time could be fully absorbed into slack. SOA 800 is chosen because this SOA is least affected by the dual-task demands and thus resembles visual search performance as single task. There are three possible scenarios: (1) In case that the set size effect at SOA 800 is smaller than the PRP effect, the hypotheses derived from the locus-of-slack method can be tested without concern. (2) In case that the set size effect at SOA 800 is larger than the PRP effect and thus larger than the duration of slack, the results should be interpreted more carefully. Depending on the amount of search time that is absorbed into slack at short SOA, a significant partial absorption of visual search time (i.e., significantly smaller set size effect at short SOA compared with the set size effect at long SOA) could still show that at least a part of visual search is performed in parallel to response selection in Task 1. (3) In case that the set size effect at SOA 800 is larger than the PRP effect and visual search time is not partially absorbed, additive effects of SOA and set size would indicate that visual search is performed after response selection has been completed. The corresponding analyses showed the following results: In Experiment 1, the PRP effect was $302 \mathrm{~ms}$. The set size effect of triple conjunction search at SOA 800 was 295 ms. In theory, visual search could have been completed during slack (295 ms < $302 \mathrm{~ms}$ ), which is why it was valid to use the locus-of-slack method. However, only $80 \mathrm{~ms}$ of visual search time were absorbed into slack at SOA 50. Considering that there was an interaction of SOA and set size in RT1 and the set size effect for RT1 at SOA 50 was also $80 \mathrm{~ms}$, we would like to argue that visual search time was probably only absorbed into slack, because participants delayed response selection in Task 1, which increased the slack time. In Experiment 2, the PRP effect was $233 \mathrm{~ms}$, but the set size effect of plus conjunction search at SOA 800 was $289 \mathrm{~ms}$. Since the set size effect exceeded the duration of slack ( $289 \mathrm{~ms}>233 \mathrm{~ms}$ ), the results should be interpreted more carefully. Visual search time was not even partially absorbed into slack as in Experiment 1, since there was no hint for a significant interaction of SOA and set size. Accordingly, plus conjunction search in Task 2 was performed after response selection in Task 1 had been completed.

The findings of Experiment 2 are in line with the results of Han (2017, Experiment 1). Han used an orientation search Task 2 that consisted of Gabor gratings. Since the stimuli differed only with respect to the orientation of the gratings but shared the same color and the same outer form, they had to be selected in a serial search process until the target grating was found. The author showed that the search task in Task 2 was performed after response selection in Task 1 had been completed. He concluded that central attention is required for serial item selection in visual search. Han's search task is comparable to the plus conjunction search Task 2 in Experiment 2. In Han's study, all Gabor gratings shared the same features, but differed only with respect to the orientation of the gratings; the same was true for plus conjunction search, as all stimuli shared the same color and form features, but differed with respect to how the features were mapped together. Accordingly, in both search tasks, none of the distractors could be ignored, so that the search process was strongly serial. For both search tasks, the results showed that visual search in Task 2 was performed only after response selection in Task 1 had been completed and central attention was available again.

The present study complements the understanding of the interplay of visual and central attention. So far, evidence regarding the dynamic of this interplay has rather been mixed, as studies showed that visual and central attention rely on distinct capacity limitations (Han, 2017, Experiment 2; Lien, Croswaite, \& Ruthruff, 2011, Experiments 1 \& 2; Pashler, 1989, 1991; Reimer \& Schubert, 2019; Reimer et al., 2015; Reimer et al., 2017), but also that they share a common capacity limitation (Brisson \& Jolicoeur, 2007a, b; Han, 2017, Experiment 1; Lien et al., 2011, Experiments 3 \& 4). Lien et al. (2011) were the first who systematically investigated which factors could influence the interplay of visual and central attention. The authors varied central attention demands by increasing the response selection difficulty in Task 1 from a two-choice to a four-choice discrimination. Their results indicated that visual search Task 2 and response selection in Task 1 were processed concurrently, when central attention demands were low (i.e., two-choice Task 1), but they were processed sequentially, when central attention demands were high (i.e., four-choice Task 1; see also Brisson \& Jolicoeur, 2007a, b). Based on these findings, Lien et al. (2011) concluded that central attention demands modulate the interplay of visual and central attention.

Following up on their conclusion, we tested in a former study whether central attention demands for response selection in Task 1 modulate the interplay of visual and central attention for a regular conjunction search Task 2 (Reimer \& Schubert, 2019). However, in our study, we did not find evidence for the influence of central attention demands for response selection in Task 1 on the interplay of visual and central attention, as the regular conjunction search Task 2 was processed in parallel to response selection in both a twochoice and a four-choice discrimination Task 1 . The results of the present study as well as an analysis of the search task in Lien et al. (2011) could reconcile the diverging findings. In the study of Lien et al. (2011), the target differed in color from the distractors, which is why it automatically captured attention. The task was not to detect the presence versus absence of the 
target, but rather the task required to identify which one of two target letters was presented. Such a task is a so-called compound search task (Müller \& Krummenacher, 2006), as it consists of two processes, a target-related and a responserelated process. Target localization required visual attention, and it seems likely that letter identification required central attention.

The critical question is whether letter identification in Lien et al.'s (2011) search Task 2 and serial item selection in regular conjunction search Task 2 in our study (Reimer \& Schubert, 2019) require similar or different amounts of central attention. Lien et al. (2011, Experiments $3 \&$ 4) found sequential performance of response selection in Task 1 and visual search in Task 2 when central attention demands in Task 1 were high, but we found concurrent performance of response selection in Task 1 and regular conjunction search in Task 2 when central attention demands in Task 1 were high. Consequently, it seems most likely that the compound search Task 2 relied more on central attention than serial item selection in the regular conjunction search Task 2. Taking the findings of the present study into account, the set size effects in both triple and plus conjunction search Tasks 2 were larger compared with the set size effects in regular conjunction search Task 2 (Reimer \& Schubert, 2019). These results are another piece of evidence that regular conjunction search Task 2 did not seem to rely as much on a serial search process, and in turn did not seem to require as much central attention for serial item selection as other conjunction search tasks. Thus, even when central attention demands in Task 1 were high, regular conjunction search in Task 2 could be performed in parallel to response selection in Task 1. However, the compound Task 2 in Lien et al. (2011) seemed to have required central attention and was performed as central attention was available again - that is, after response selection in Task 1 had been completed. To summarize, the described studies could be interpreted as showing that a visual search task can indeed require central attention, either for serial item selection and feature binding as in conjunction search (the present study), or for the response-related process in compound search (Brisson \& Jolicoeur, 2007a, b; Lien et al., 2011), or both (Han, 2017). Depending on the involvement of central attention in visual search, visual search in Task 2 and response selection in Task 1 can still be processed in parallel if central attention demands are low, but they are processed sequentially if central attention demands are high.

\section{Conclusion}

To conclude, the present study complements previous research on the interplay of visual and central attention by demonstrating that visual search demands modulate this interplay (see also Han, 2017). More concretely, the findings provide new insights into the interplay of visual and central attention for triple and plus conjunction search Tasks 2 compared with regular conjunction search Tasks 2 (Reimer \& Schubert, 2019; Reimer et al., 2015). Overall, visual and central attention rely on distinct capacity limitations for regular conjunction search Tasks 2, but they share a common capacity limitation for triple and plus conjunction search Tasks 2 , as these search tasks rely strongly on serial item selection and feature binding.

Funding This work was funded by the German Research Foundation (DFG, grants Schu 1397/5-2 and 1397/7-1 awarded to Torsten Schubert).

\section{Compliance with ethical standards}

Conflict of interest The authors declare that they have no conflict of interest.

Ethical approval All procedures performed in all two experiments were in accordance with the ethical standards of the ethics committee of the department of psychology of the Humboldt-Universität zu Berlin and with the 1964 Helsinki declaration and its later amendments.

Informed consent Written informed consent was obtained from all individual participants included in the study.

\section{References}

Bichot, N. P., Rossi, A. F., \& Desimone, R. (2005). Parallel and serial neural mechanisms for visual search in macaque area V4. Science, 308(5721), 529-534. doi:https://doi.org/10.1126/science.1109676

Brisson, B., \& Jolicoeur, P. (2007a). A psychological refractory period in access to visual short-term memory and the deployment of visualspatial attention: Multitasking processing deficits revealed by eventrelated potentials. Psychophysiology, 44(2), 323-333. doi:https:// doi.org/10.1111/j.1469-8986.2007.00503.x

Brisson, B., \& Jolicoeur, P. (2007b). Electrophysiological evidence of central interference in the control of visuospatial attention. Psychonomic Bulletin \& Review, 14(1), 126-132. doi:https://doi. org/10.3758/BF03194039

Carrasco, M. (2011). Visual attention: The past 25 years. Vision Research, 51(13), 1484-1525. doi:https://doi.org/10.1016/j.visres.2011.04. 012

Di Lollo, V. (2012). The feature-binding problem is an ill-posed problem. Trends in Cognitive Sciences, 16(6), 317-321. doi:https://doi.org/ 10.1016/j.tics.2012.04.007

Duncan, J., \& Humphreys, G. W. (1989). Visual search and stimulus similarity. Psychological Review, 96(3), 433-458. doi:https://doi. org/10.1037/0033-295X.96.3.433

Duncan, J., \& Humphreys, G. W. (1992). Beyond the search surface: Visual search and attentional engagement. Journal of Experimental Psychology: Human Perception and Performance, 18(2), 578-588. doi:https://doi.org/10.1037/0096-1523.18.2.578

Eckstein, M. P. (2011). Visual search: A retrospective. Journal of Vision, 11(5):14, 1-36. doi:https://doi.org/10.1167/11.5.14

Eimer, M. (2015). EPS Mid-Career Award 2014: The control of attention in visual search - Cognitive and neural mechanisms. The Quarterly Journal of Experimental Psychology, 68(12), 2437-2463. doi: https://doi.org/10.1080/17470218.2015.1065283

Fischer, R., \& Plessow, F. (2015). Efficient multitasking: Parallel versus serial processing of multiple tasks. Frontiers in Psychology, 6(1366). doi:https://doi.org/10.3389/fpsyg.2015.01366 
Han, S. W. (2017). The involvement of central attention in visual search is determined by task demands. Attention, Perception, \& Psychophysics, 79(3), 726-737. doi:https://doi.org/10.3758/ s13414-017-1278-9

Huang, L., \& Pashler, H. (2007). A Boolean map theory of visual attention. Psychological Review, 114(3), 599-631. doi:https://doi.org/10. 1037/0033-295X.114.3.599

Huestegge, L., \& Koch, I. (2010). Crossmodal action selection: Evidence from dual-task compatibility. Memory \& Cognition, 38(4), 493-501. doi:https://doi.org/10.3758/MC.38.4.493

Humphreys, G. W., Hodsoll, J., Olivers, C. N. L., \& Yoon, E. Y. (2006). Contributions from cognitive neuroscience to understanding functional mechanisms of visual search. Visual Cognition, 14(4/8), 832850. doi:https://doi.org/10.1080/13506280500195516

Kirk, R. E. (2013). Experimental design: Procedures for the behavioral sciences (4th ed.). Thousand Oaks, CA: SAGE Publications.

Lien, M.-C., Croswaite, K., \& Ruthruff, E. (2011). Controlling spatial attention without central attentional resources: Evidence from event-related potentials. Visual Cognition, 19(1), 37-78. doi: https://doi.org/10.1080/13506285.2010.491643

Logan, G. D., \& Gordon, R. D. (2001). Executive control of visual attention in dual-task situations. Psychological Review, 108(2), 393-434. doi:https://doi.org/10.1037/0033-295X.108.2.393.

Meyer, D. E., \& Kieras, D. E. (1997). A computational theory of executive cognitive processes and multiple-task performance: Part 1. Basic mechanisms. Psychological Review, 104(1), 3-65. doi: https://doi.org/10.1037/0033-295X.104.1.3

Müller, H. J., \& Krummenacher, J. (2006). Visual search and selective attention. Visual Cognition, 14(4/8), 389-410. doi:https://doi.org/ $10.1080 / 13506280500527676$

Navon, D., \& Miller, J. (2002). Queuing or sharing? A critical evaluation of the single-bottleneck notion. Cognitive Psychology, 44(3), 193251. doi:https://doi.org/10.1006/cogp.2001.0767

Pashler, H. (1989). Dissociations and dependencies between speed and accuracy: Evidence for a two-component theory of divided attention in simple tasks. Cognitive Psychology, 21(4), 469-514. doi:https:// doi.org/10.1016/0010-0285(89)90016-9

Pashler, H. (1991). Shifting visual attention and selecting motor responses: Distinct attentional mechanisms. Journal of Experimental Psychology: Human Perception and Performance, 17(4), 10231040. doi:https://doi.org/10.1037/0096-1523.17.4.1023

Pashler, H. (1994). Dual-task interference in simple tasks: Data and theory. Psychological Bulletin, 116(2), 220-244. doi:https://doi.org/10. 1037/0033-2909.116.2.220

Pashler, H., \& Johnston, J. C. (1989). Chronometric evidence for central postponement in temporally overlapping tasks. The Quarterly Journal of Experimental Psychology A: Human Experimental Psychology, 41(1-A), 19-45. doi:https://doi.org/10.1080/ 14640748908402351

Reimer, C. B., \& Schubert, T. (2019). More insight into the interplay of response selection and visual attention in dual-tasks: Masked visual search and response selection are performed in parallel. Psychological Research, 83(3), 459-475. doi:https://doi.org/10. 1007/s00426-017-0906-2

Reimer, C. B., Strobach, T., Frensch, P. A., \& Schubert, T. (2015). Are processing limitations of visual attention and response selection subject to the same bottleneck in dual-tasks? Attention, Perception, \& Psychophysics, 77(4), 1052-1069. doi:https://doi.org/10.3758/ s13414-015-0874-9

Reimer, C. B., Strobach, T., \& Schubert, T. (2017). Concurrent deployment of visual attention and response selection bottleneck in a dualtask: Electrophysiological and behavioural evidence. The Quarterly Journal of Experimental Psychology, 70(12), 2460-2477. doi: https://doi.org/10.1080/17470218.2016.1245348

Schubert, T. (1999). Processing differences between simple and choice reactions affect bottleneck localization in overlapping tasks. Journal of Experimental Psychology: Human Perception and Performance, 25(2), 408-425. doi:https://doi.org/10.1037/0096-1523.25.2.408

Schubert, T. (2008). The central attentional limitation and executive control. Frontiers in Bioscience, 13(13), 3569-3580. doi:https://doi.org/ $10.2741 / 2950$

Schubert, T., Fischer, R., \& Stelzel, C. (2008). Response activation in overlapping tasks and the response-selection bottleneck. Journal of Experimental Psychology: Human Perception and Performance, 34(2), 376-397. doi:https://doi.org/10.1037/0096-1523.34.2.376

Schweickert, R. (1978). A critical path generalization of the additive factor method: Analysis of a Stroop task. Journal of Mathematical Psychology, 18(2), 105-139. doi:https://doi.org/10.1016/00222496(78)90059-7

Schweickert, R. (1980). Critical-path scheduling of mental processes in a dual task. Science, 209(4457), 704-706. doi:https://doi.org/10.1126/ science. 7394529

Sigman, M., \& Dehaene, S. (2006). Dynamics of the central bottleneck: Dual-task and task uncertainty. PLoS Biology, 4(7), e220. doi:https:// doi.org/10.1371/journal.pbio.0040220

Strobach, T., Schütz, A., \& Schubert, T. (2015). On the importance of Task 1 and error performance measures in PRP dual-task studies. Frontiers in Psychology, 6, 403. doi:https://doi.org/10.3389/fpsyg. 2015.00403

Sung, K. (2008). Serial and parallel attentive visual searches: Evidence from cumulative distribution functions of response times. Journal of Experimental Psychology: Human Perception and Performance, 34(6), 1372-1388. doi:https://doi.org/10.1037/a0011852

Thornton, T. L., \& Gilden, D. L. (2007). Parallel and serial processes in visual search. Psychological Review, 114(1), 71-103. doi:https://doi. org/10.1037/0033-295X.114.1.71

Tombu, M., \& Jolicoeur, P. (2003). A central capacity sharing model of dual-task performance. Journal of Experimental Psychology: Human Perception and Performance, 29(1), 3-18. doi:https://doi. org/10.1037/0096-1523.29.1.3

Townsend, J. T. (1971). A note on the identifiability of parallel and serial processes. Perception \& Psychophysics, 10(3), 161-163. doi:https:// doi.org/10.3758/BF03205778

Treisman, A. (1988). Features and objects: The Fourteenth Bartlett Memorial Lecture. The Quarterly Journal of Experimental Psychology Section A, 40(2), 201-237. doi:https://doi.org/10.1080/ 02724988843000104

Treisman, A. (1996). The binding problem. Current Opinion in Neurobiology, 6(2), 171-178. doi:https://doi.org/10.1016/S09594388(96)80070-5

Treisman, A. (1998). Feature binding, attention and object perception. Philosophical Transactions of the Royal Society B, 353(1373), 1295-1306. doi:https://doi.org/10.1098/rstb.1998.0284

Treisman, A. (2006). How the deployment of attention determines what we see. Visual Cognition, 14(4/8), 411-443. doi:https://doi.org/10. 1080/13506280500195250

Treisman, A., \& Gelade, G. (1980). A feature integration theory of attention. Cognitive Psychology, 12(1), 97-136. doi:https://doi.org/10. 1016/0010-0285(80)90005-5

Treisman, A., \& Sato, S. (1990). Conjunction search revisited. Journal of Experimental Psychology: Human Perception and Performance, 16(3), 459-478. doi:https://doi.org/10.1037/0096-1523.16.3.459

Welford, A. T. (1952). The psychological refractory period and the timing of high speed performance-A review and a theory. British Journal of Psychology, 43(1), 2-19. doi:https://doi.org/10.1111/j.2044-8295. 1952.tb00322.x

Wolfe, J. M. (1994). Guided search 2.0. A revised model of visual search. Psychonomic Bulletin \& Review, 1(2), 202-238. doi:https://doi.org/ 10.3758/BF03200774

Wolfe, J. M. (1998). What can 1 million trials tell us about visual search? Psychological Science, 9(1), 33-39. doi:https://doi.org/10.1111/ 1467-9280.00006 
Wolfe, J. M. (2007). Guided Search 4.0: Current progress with a model of visual search. In W. Gray (Ed.), Integrated models of cognitive system (pp. 99-119). New York, NY: Oxford University Press.

Wolfe, J. M. (2012). The binding problem lives on: Comment on Di Lollo. Trends in Cognitive Sciences, 16(6), 307-308. doi:https:// doi.org/10.1016/j.tics.2012.04.013

Wolfe, J. M., \& Bennett S. C. (1997). Preattentive object files: Shapeless bundles of basic features. Vision Research, 37(1), 25-43. doi:https:// doi.org/10.1016/S0042-6989(96)00111-3

Wolfe, J. M., Cave, K. R., \& Franzel, S. L. (1989). Guided search: An alternative to the feature integration model for visual search. Journal of Experimental Psychology: Human Perception and Performance, 15(3), 419-433. doi:https://doi.org/10.1037/0096-1523.15.3.419

Wolfe, J. M., Palmer, E. M., \& Horowitz, T. S. (2010). Reaction time distributions constrain models of visual search. Vision Research, 50(14), 1304-1311. doi:https://doi.org/10.1016/j.visres.2009.11. 002

Publisher's note Springer Nature remains neutral with regard to jurisdictional claims in published maps and institutional affiliations. 